\title{
Combined effect of Helicobacter pylori infection and elevated C-reactive protein on 3-month prognosis of ischemic stroke
}

\author{
Tan Xu, Chenhuan Zhang, Aili Wang, Yonghong Zhang \\ School of Public Health, Medical College of Soochow University, P. R. China
}

Objective: To investigate the combined effect of Helicobacter pylori (HP) infection and elevated C-reactive protein (CRP) on the prognosis of acute ischemic stroke patients at 3 months. Methods: From June 2011 to December 2013, 1110 confirmed cases of ischemic stroke were included from 14 hospitals in China who were followed at prospective cohort study. Serum Helicobacter pylori immunoglobulin G (HP-lgG) and CRP were tested at cases admission. Outcomes were defined as death or cardiovascular events at 3 months after ischemic stroke onset. According to HP-IgG and CRP levels, all patients were divided into four groups: G1 was low-level HP-lgG/ low-level CRP, G2 was low-level HP-lgG/ CRP elevated, G3 was HP-lgG elevated/ low-level CRP and G4 was elevated HP-lgG/ elevated CRP. With $\mathrm{G} 1$ as a reference, logistic regression analysis was used to analyze the relationship between HP-IgG elevation with CRP elevation and the poor prognosis of ischemic stroke at 3 months respectively, and the combined effect of elevated HP-IgG with elevated CRP on poor prognosis of ischemic stroke for 3 months. Results: Within 3 months after ischemic stroke onset, a total of $38(3.42 \%)$ patients died and $76(6.85 \%)$ patients died or had cardiovascular events. After multifactorial adjustment, patients with elevated HP-IgG at 3 months had a 3.14 -fold $(95 \%$ $\mathrm{Cl}: 1.30 \sim 7.57)$ and 2.52 -fold $(95 \% \mathrm{Cl}: 1.45 \sim 4.36)$ risk on death and death or cardiovascular events compared with patients with low-level HP-lgG. Patients with elevated CRP had a 3.00 -fold (95\% Cl: $1.40 \sim 6.43)$ and 1.65-fold (95\% Cl: $0.91 \sim 2.99)$ risk of death and death or cardiovascular events at 3 months compared with patients with low CRP. With multifactorial adjustment, combined effects of elevated HP-lgG and elevated CRP was found that G4 patients had a 10.00 -fold risk $(95 \% \mathrm{Cl}$ : 2.85 35.05) of death at 3 months and 4.24-fold (95\% Cl: $1.83 \sim 9.85)$ risk of death or cardiovascular events compared with G1 patients. Conclusion: HP infection and elevated CRP have a combined effect on poor prognosis of stroke.

Table 1. ORs of HP infection and elevated CRP for the prognosis of stroke.

\begin{tabular}{|c|c|c|c|c|}
\hline & \multicolumn{2}{|c|}{ unadjusted } & \multicolumn{2}{|c|}{ adjusted } \\
\hline & $\mathrm{OR}(95 \% \mathrm{Cl})$ & $\mathrm{P}$ & $\mathrm{OR}(95 \% \mathrm{Cl})$ & $\mathrm{P}$ \\
\hline \multicolumn{5}{|l|}{ Death } \\
\hline HP infection ${ }^{a}$ & $3.91(1.78-8.60)$ & $<0.001$ & $3.14(1.30-7.57)$ & 0.011 \\
\hline CRP elevated & $5.10(2.63-9.88)$ & $<0.001$ & $3.00(1.40-6.43)$ & 0.005 \\
\hline \multicolumn{5}{|c|}{ Death or vascular events } \\
\hline HP infectiona & $2.61(1.57-4.35)$ & $<0.001$ & $2.52(1.45-4.36)$ & 0.001 \\
\hline CRP elevated & $2.56(1.51-4.33)$ & $<0.001$ & $1.65(0.91-2.99)$ & 0.098 \\
\hline
\end{tabular}

aThe adjusted factors were age, sex, smoking, drinking, NIHSS score, diastolic pressure, fasting blood sugar, total cholesterol, white blood cell count, history of coronary heart disease, history of diabetes mellitus, history of hypertension and CRP; bThe adjustment factors were age, sex, smoking, drinking, NIHSS score, diastolic pressure, fasting blood sugar, total cholesterol, white blood cell count, history of coronary heart disease, history of diabetes mellitus, history of hypertension and HP IgG。

Table 2. Combined effects of HP infection and elevated CRP on the prognosis of stroke.

\begin{tabular}{cllll}
\hline & \multicolumn{2}{c}{ unadjusted } & \multicolumn{1}{c}{ adjusted $^{\mathrm{a}}$} \\
\cline { 2 - 5 } & $\mathrm{OR}(95 \% \mathrm{Cl})$ & $\mathrm{P}$ & OR(95\%Cl) & $\mathrm{P}$ \\
\hline Death & & & 1.00 & \\
G1 & 1.00 & 0.017 & $3.85(0.86-17.18)$ & 0.077 \\
G2 & $5.51(1.35-22.47)$ & 0.010 & $3.58(1.14-11.27)$ & 0.029 \\
G3 & $4.26(1.42-12.75)$ & $<0.001$ & $10.00(2.85-35.05)$ & $<0.001$ \\
G4 & $23.89(7.55-75.52)$ & & & \\
Death or vascular events & 1.00 & & 1.00 & \\
G1 & $2.06(0.78-5.43)$ & 0.142 & $1.67(0.60-4.62)$ & 0.324 \\
G2 & $2.43(1.34-4.42)$ & 0.004 & $2.47(1.32-4.64)$ & 0.005 \\
G3 & $7.52(3.57-15.81)$ & $<0.001$ & $4.24(1.83-9.85)$ & $<0.001$ \\
G4 & & & \\
\hline
\end{tabular}

a The adjusting factors were age, sex, smoking, drinking, NIHSS score, diastolic pressure, fasting blood sugar, total cholesterol, white blood cell count, history of coronary heart disease, diabetes mellitus and hypertension. G1 was low-level HP-lgG/low-level CRP, G2 was low-level HP-lgG/CRP elevated, G3 was HP-lgG elevated/low-level CRP, and G4 was HP-lgG elevated/CRP elevated. 